\title{
The bright optical flash from GRB 060117 ${ }^{\star}$
}

\author{
M. Jelínek ${ }^{1}$, M. Prouza ${ }^{2,6}$, P. Kubánek ${ }^{3,4}$, R. Hudec ${ }^{3}$, M. Nekola ${ }^{3}$, J. Řídký2,6, J. Grygarar,6, M. Boháčováá,6, \\ A. J. Castro-Tirado ${ }^{1}$, J. Gorosabel ${ }^{1}$, M. Hrabovský ${ }^{2,6}$, D. Mandát ${ }^{2,6}$, D. Nosek ${ }^{5,6}$, L. Nožka ${ }^{2,6}$, M. Palatka ${ }^{2,6}$, \\ S. B. Pandey ${ }^{1}$, M. Pech ${ }^{2,6}$, P. Schovánek ${ }^{2,6}$, R. Šmída ${ }^{2,6}$, P. Trávníček $^{2,6}$, A. de Ugarte Postigo ${ }^{1}$, and S. Vítek ${ }^{1}$ \\ 1 Instituto de Astrofísica de Andalucía (IAA-CSIC), Apartado de Correos, 3.004, 18080 Granada, Spain \\ e-mail: mates@iaa.es \\ 2 Fyzikální ústav AV ČR, Na Slovance 2, 18221 Praha 8, Czech Republic \\ 3 Astronomický ústav AV ČR, Fričova 298, 25165 Ondřejov, Czech Republic \\ 4 INTEGRAL Science Data Centre, Ch. d'Ecogia 16, 1290 Versoix, Switzerland \\ 5 Ústav částicové a jaderné fyziky, MFF UK Praha, V Holešovičkách 2, 18000 Praha 8, Czech Republic \\ ${ }^{6}$ For the Pierre Auger Collaboration
}

Received 26 February 2006 / Accepted 30 May 2006

\section{ABSTRACT}

\begin{abstract}
We present a discovery and observation of an extraordinarily bright prompt optical emission of the GRB 060117 obtained by a widefield camera atop the robotic telescope FRAM of the Pierre Auger Observatory from 2 to 10 min after the GRB. We found rapid average temporal flux decay of $\alpha=-1.7 \pm 0.1$ and a peak brightness $R=10.1$ mag. Later observations by other instruments set a strong limit on the optical and radio transient fluxes, unveiling an unexpectedly rapid further decay. We present an interpretation featuring a relatively steep electron-distribution parameter $p \simeq 3.0$ and providing a straightforward solution for the overall fast decay of this optical transient as a transition between reverse and forward shock.
\end{abstract}

Key words. gamma-rays: bursts

\section{Introduction}

After the establishment of the GRB Coordinates Network (GCN) (Barthelmy et al. 1995) in 1995, the technical advances have enabled a fast and reliable dissemination of satellite gamma-ray burst (GRB) data to ground-based observers. Subsequently, wide use of the sophisticated robotic follow-up systems has led to the first insight into the very early phases (less than 5 min after trigger) of the optical transients (OTs) accompanying some GRBs. However, despite the extended efforts, optical data at the very early stages are still sparse, so the whole picture remains unclear.

The definition of the prompt optical emission was given by Piran (1999) as the optical emission arising during the $\gamma$-emission period. This early emission is usually explained in terms of either a reverse or an internal shock (Sari \& Piran 1999). With a certain delay after the GRB, the afterglow - emission due to the forward shocks - starts to dominate the steeply decaying prompt emission. This transition flattens the lightcurve. The original rapid decay of the prompt emission slows down to a more modest decay due to the afterglow. It is generally accepted that the physical mechanisms of the prompt emission and the afterglow are distinct. The distinction is probably not absolute, some observations suggest (cf. Chincarini et al. 2005) that at least the $X$-ray intensity of the early emission phases can be extrapolated from the GRB emission itself.

^ Based on observations of robotic telescope FRAM, operated by the Pierre Auger collaboration at Los Leones site, Malargüe, Argentina.
In this letter we present the observation of a very bright optical transient associated with GRB 060117 observed by the robotic telescope FRAM.

\section{Observations}

A bright long-soft GRB 060117 was detected by Swift satellite on January 17, 2006, at 6:50:01.6UT. It showed a multi-peak structure with $T_{90}=16 \pm 1 \mathrm{~s}$ with maximum peak flux $48.9 \pm$ $1.6 \mathrm{ph} \mathrm{cm}^{-2} \mathrm{~s}^{-1}$. Coordinates computed by Swift were available within $19 \mathrm{~s}$ and immediately distributed by GCN (Cummings et al. 2006).

FRAM received the notice at 06:50:20.8 UT, 19.2 s after the trigger and immediately started the slew. The first exposure started at 06:52:05.4, 123.8 s after the GRB. Eight images with different exposures were taken before the observation was terminated. A bright, rapidly decaying object was found, and its presence was reported by Kubánek et al. (2006) and Jelínek et al. (2006) soon after the discovery. The point-spread function of the object is similar to the stars in the image, and the object did not move more than $2^{\prime \prime}$ over the course of our observation, ruling out a near-Earth object crossing the field of view. The weather conditions during the observation were very good, but the Moon was nearly full (93\%) and the GRB location was only slightly more than $5^{\circ}$ above the horizon. Consequently, the magnitude limits of our observation were $\sim 2.5$ mag worse than the technical limit of the FRAM instrument in the optimal conditions. Table 1 displays the log of our observations (see also Fig. 1), where the magnitude errors do not include systematic error of the USNO-B1.0 R1 magnitude, which should be $<0.1 \mathrm{mag}$. 


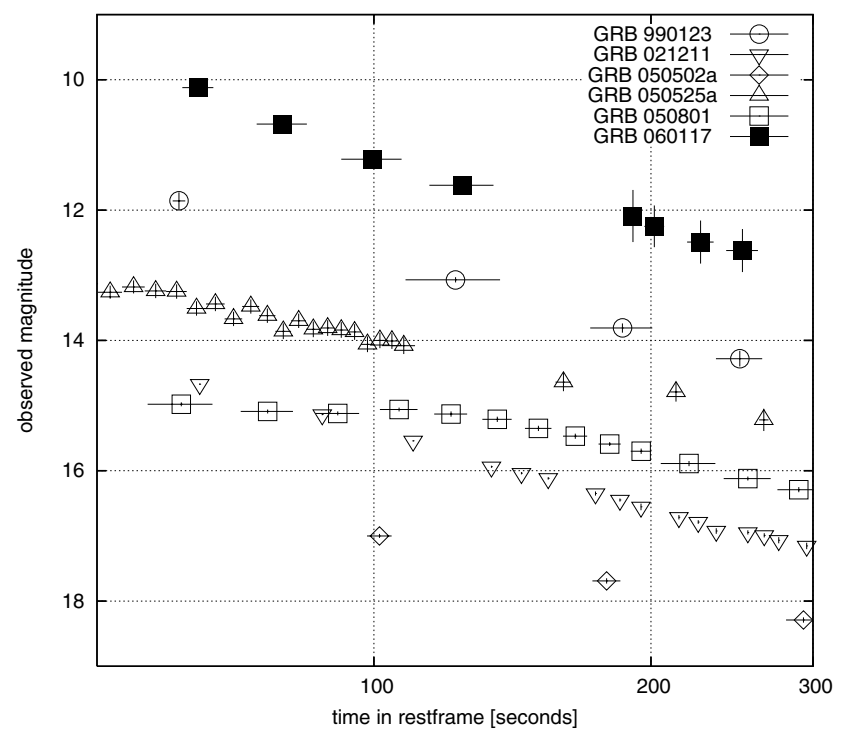

Fig. 1. The optical light curve of GRB 060117 in comparison with other well-covered early GRB optical emissions: GRB 990123 (Akerlof et al. 1999), GRB 021211 ( $\mathrm{Li}$ et al. 2003), GRB 050502a (Guidorzi et al. 2005), GRB 050525a (Blustin et al. 2006), and GRB 050801 (Rykoff et al. 2006). In this figure we use $z \approx 1.0$ for GRB 060117. Observed $R$-band magnitudes are shown, except for GRB 050525a, where the $V$-band values are plotted.

Table 1. Optical $R$-band photometric observations of the optical flash GRB 060117. The magnitudes are not corrected for Galactic extinction $\left(A_{R} \sim 0.01 \mathrm{mag}\right) . T-T_{0}$ is mean exposure time since the GRB.

\begin{tabular}{lcccc}
\hline \hline UT Date of exp. start & $T-T_{0}[\mathrm{~s}]$ & $T_{\exp }[\mathrm{s}]$ & $R$ & $\delta R$ \\
\hline 2006 Jan. 17.786169 & 128.8 & 10 & 10.12 & 0.13 \\
2006 Jan. 17.786833 & 159.1 & 20 & 10.68 & 0.12 \\
2006 Jan. 17.786343 & 199.3 & 30 & 11.22 & 0.14 \\
2006 Jan. 17.787583 & 249.7 & 40 & 11.62 & 0.18 \\
2006 Jan. 17.789109 & 382.4 & 10 & 12.09 & 0.45 \\
2006 Jan. 17.789410 & 403.4 & 20 & 12.25 & 0.36 \\
2006 Jan. 17.789815 & 452.9 & 30 & 12.49 & 0.37 \\
2006 Jan. 17.790336 & 502.9 & 40 & 12.62 & 0.37 \\
\hline
\end{tabular}

An optical counterpart to GRB 060117 was found $128.8 \mathrm{~s}$ after the burst at

$\alpha=21^{\mathrm{h}} 51^{\mathrm{m}} 36.23 \quad \delta=-59^{\circ} 58^{\prime} 39^{\mathrm{s}} \cdot 3 \quad \pm 1^{\prime \prime} \cdot 5 \quad(\mathrm{~J} 2000)$.

The error amounts to a $1-\sigma$ uncertainty including systematic errors.

\section{Follow-up}

Swift itself could not observe the GRB with its X-ray and optical instruments, because of the Sun observing constraint (Campana et al. 2006). One month later on Feb. 14 and 15, 2006, Swift XRT (Burrows et al. 2005) pointed to the burst position and did not detect any source at the corresponding position with a 3- $\sigma$ limit of $1.0 \times 10^{-3}$ counts $\mathrm{s}^{-1}$, corresponding to an unabsorbed $0.2-10 \mathrm{keV}$ flux upper limit of $2.3 \times 10^{-14} \mathrm{erg} \mathrm{cm}^{-2} \mathrm{~s}^{-1}$. The burst was also detected by Konus-Wind (Golenetskii et al. 2006) and by Suzaku WAM (Terada et al. 2006).

Unfortunately, the later optical follow-up was unsuccessful due to cloudy weather in both New Zealand and South Africa. The limits reported by PROMPT (Nysewander et al. 2006) (observations beginning $18.0 \mathrm{~h}$ after the burst), however, suggest a surprisingly rapid decay. The search for a radio afterglow was also unsuccessful (Schmidt et al. 2006).
The lag-luminosity pseudo-redshift estimation from Swift data yields $z \simeq 1.3 \pm 0.3$ (Cummings et al. 2006). The redshift estimate based on the $\gamma$-ray data from Konus-Wind gives $z \simeq 0.45 \pm 0.2$ (Pelangeon \& Atteia 2006).

\section{Data acquisition and reduction}

FRAM is part of the Pierre Auger cosmic-ray observatory (Pierre Auger Collaboration 2005), and its main purpose is to immediately monitor the atmospheric transmission. FRAM works as an independent, RTS2-driven (Kubánek et al. 2004), fully robotic system, and it performs a photometric calibration of the sky on various UV-to-optical wavelengths using a $0.2 \mathrm{~m}$ telescope and a photoelectric photomultiplier. As a primary objective, FRAM observes a set of chosen standard stars and a terrestrial light source. From these observations it obtains instant extinction coefficients and the extinction wavelength dependence. Additionally, FRAM is able to follow GCN alerts, using its wide-field camera with a fixed $R$-band filter.

The wide-field camera consists of a Carl Zeiss Sonnar $200 \mathrm{~mm} f / 2.8$ telephoto lens, SBIG ST7 imager, and Bessel $R$-band filter. The ST7 camera has a $768 \times 512$ Kodak KAF$0402 \mathrm{E} C \mathrm{CD}$ that covers a field of view of $120^{\prime} \times 80^{\prime}$ with a scale of 9.'6/pixel. The effective diameter of the lens is $57 \mathrm{~mm}$ and the $3 \sigma$ limiting magnitude under optimum conditions reaches $R \sim 15.0$ for a $30 \mathrm{~s}$ exposure.

The raw images were dark-frame subtracted using a median of several dark-frame exposures. Given the significant dark current of the camera, the darks were treated separately for each exposure time. The flat-field correction was then applied using the median of 40 normalized $1 \mathrm{~s}$ exposures obtained while mosaicing through the twilight sky. The aperture photometry was done using the phot routine in IRAF $^{1}$ with the aperture diameter of 2 pixels. To get a precise astrometric position of the source, we used the four most significant images and computed the average position.

The images were astrometrically and photometrically calibrated on the fly using the past program in the context of JIBARO (de Ugarte Postigo et al. 2005), using all sources with more than 10- $\sigma$ significance from the image compared to USNO-B1.0 (Monet et al. 1998) positions and $R 1$ magnitudes. Past employs a sigma-clipped third-degree polynomial surface fit. For the astrometry, an error-weighted mean of the zero point is used. Systematic errors of USNO-B1.0 should be less than 0.2 in the astrometry and less than 0.1 mag for the photometry. Since the Galactic extinction, taken from the maps published by Schlegel et al. (1998) is very low $\left(E_{B-V}=0.038\right)$, we neglect this value in the following discussion.

\section{Discussion}

In the search for the interpretation of the lightcurve, we assume a uniform ISM, and that the influence of the internal shock emission on the lightcurve is negligible because our observation starts $\sim 100$ s after the end of the gamma-ray burst.

In the simplest case, the lightcurve can be fitted and a single power law with a temporal flux-decay index $\alpha=-1.73 \pm 0.12$. The $\chi^{2} /$ d.o.f. for this fit is 1.296 . If we assume this decay to be a signature of a pure forward shock, we get the value of the

1 IRAF is distributed by the National Optical Astronomy Observatory, which is operated by the Association of Universities for Research in Astronomy, Inc., under co-operative agreement with the National Science Foundation. 

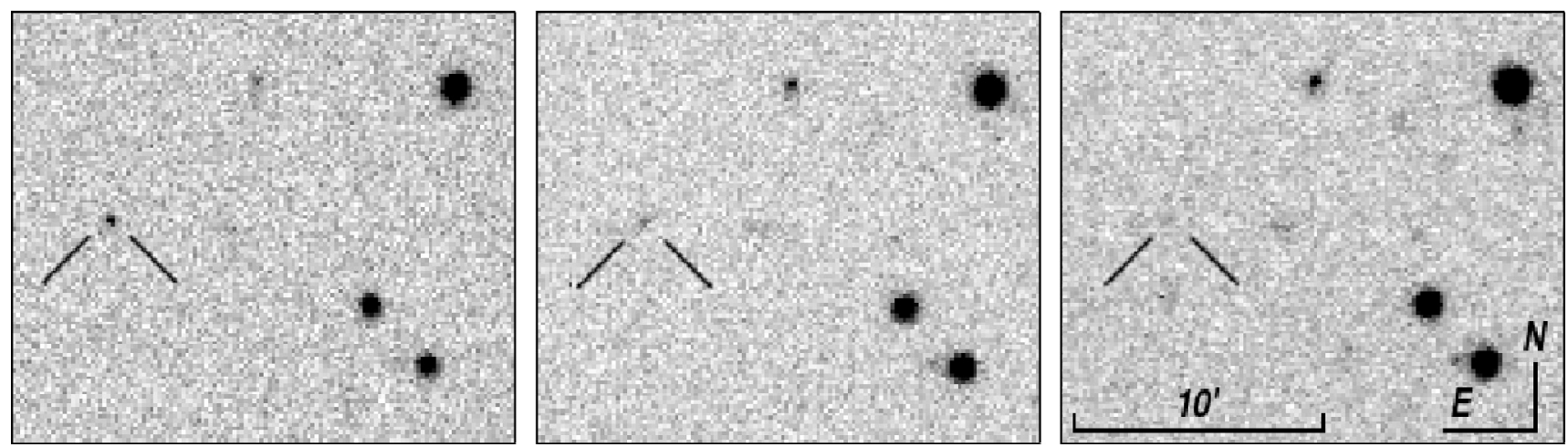

Fig. 2. Details of the surroundings of the optical flash of GRB 060117 as observed by FRAM. Images taken 129, 249, and 480 s after the trigger.

electron energy distribution power-law index $p=3.3 \pm 0.1$. This value is very high in comparison with other known optical transients, but it is consistent with $p=3.82_{-0.5}^{+1.0}$ as computed from the Konus-Wind spectra of the GRB (Golenetskii et al. 2006) (cf. Shen et al. 2005). In contrast, if the linear decay is a signature of a pure reverse shock, we get $p=2.0 \pm 0.1$ - close to the typical value for the optical transients observed so far. We should note that such a reverse shock emission should be accompanied by a forward shock with $\alpha_{\mathrm{F}} \simeq-0.7$.

Another possibility (after Shao \& Dai 2005) is to interpret the data as a type I lightcurve (as given by Zhang et al. 2003), which depicts a transition between the reverse and the forward shock with the passage of the typical frequency break $v_{m}$ through the observed passband at time $t_{m, f}$. We assume the lightcurve is initially dominated by a rapidly falling reverse-shock emission with $F_{v, r} \sim t_{d}^{-(27 p+7) / 35}$, followed by a forward-shock emission that rises as $F_{v, r} \sim t_{d}^{+1 / 2}$ before $t_{m, f}$ and then decays with $F_{v, f} \sim$ $t_{d}^{-(3 p-3) / 4}$. Using a $\chi^{2}$ minimization fit to this scenario, we obtain $p=2.96 \pm 0.06$, a magnitude of forward shock maxima $m_{m, f}=$ $11.82 \pm 0.04$, a time of the maxima $t_{m, f}=301 \pm 4 \mathrm{~s}$ (after trigger), and a magnitude of the reverse shock at $t=t_{m, f} m_{m, r}=12.43 \pm$ 0.05 ( $\chi^{2} /$ d.o.f. for this fit is 0.015$)$. Corresponding decay indices are $\alpha_{\mathrm{R}}=2.49 \pm 0.05$ and $\alpha_{\mathrm{F}}=1.47 \pm 0.03$ (see Fig. 3). If the crossing time $t_{\times}$(Zhang et al. 2003) coincides with the end of the GRB (i.e. $\sim 20 \mathrm{~s}$ ), then we can estimate the peak magnitude of this OT as $R \sim 5$ mag by backward extrapolation.

Note, that this is only one of the plausible interpretations. There may be other possible explanations for this behaviour including density jump in the media (Lazzati et al. 2002) or energy injection (Björnsson et al. 2004). Without a multiwavelength observation it is impossible to distinguish which of these possibilities actually took place.

The position of the burst and its distance from the Sun made the object difficult to observe. PROMPT (Nysewander et al. 2006) shows that the bright OT decayed very fast, and 20 hours after the burst its magnitude was already $I>21.2$. Using the procedure of Šimon et al. (2001) we transform this limit to the filter of our observation: $R \gtrsim 21.5$ From this limit we then get the estimate of an average late decay as $\alpha_{\text {late }}<-1.62$. Among the three interpretations we have shown, only the pure forward shock scenario is compatible with this limit without introducing an unusually early jet break, which is required to explain the other two scenarios.

Soon after the Swift trigger, a suspicion of an extremely lowredshift GRB was raised (Tanvir 2006) due to the presence of a nearby galaxy PGC 128172 with $z=0.04$ in the Swift errorbox. However, this discovered transient lies 3!'1 from this galaxy, accordingly the projected distance $-160 \mathrm{kpc}-$ is approximately

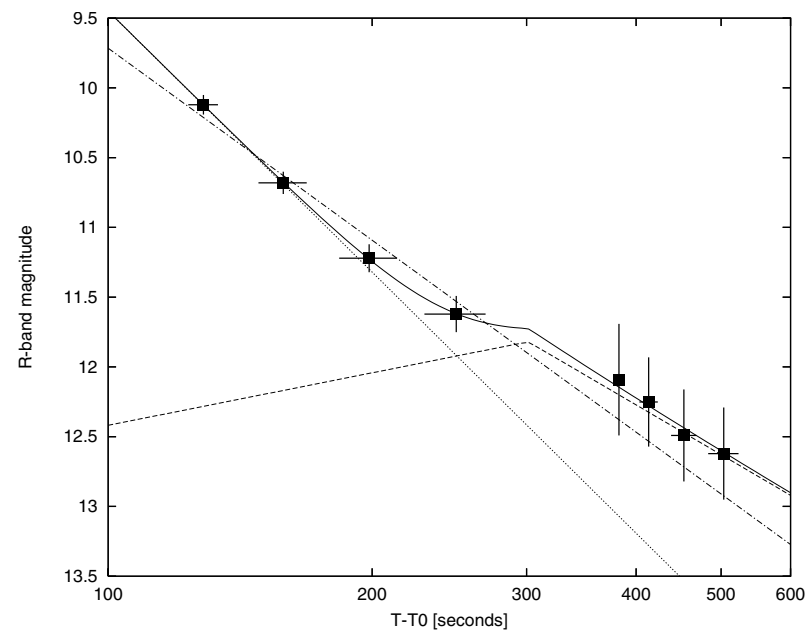

Fig. 3. The $R$-band afterglow lightcurve of GRB 060117. The lightcurve is fitted as a superposition of reverse shock (dotted line) and forward shock (dashed line). The linear fit is plotted by a dot-and-dash line.

four times larger than the visible major diameter of this galaxy. Furthermore, the position angle of the transient with respect to the PGC 128172, which we observe practically edge-on, is $97^{\circ}$. The association of the OT with this galaxy is, therefore, quite unlikely.

\section{Conclusions}

The GRB 060117 is the most intense (in terms of peak flux) GRB detected so far by Swift. With the maximum brightness of $R=$ $10.1 \mathrm{mag}$, FRAM has discovered one of the optically brightest prompt optical emissions ever detected. The initial optical decay was found to be one of the steepest of an early GRB optical afterglow observed.

We have presented 3 scenarios for explaining the lightcurve. The apparent change in its slope is neglected in two simple scenarios, where we suppose the observed lightcurve to only be the trace of a forward, resp. reverse, shock. In the third (preferred) scenario, the shape of the lightcurve is explained as a transition between reverse and forward shock emission. The forwardshock-only interpretation is flawless regarding the PROMPT limit, but shows rather spurious value of $p$. The other two scenarios (i.e. those involving reverse shock) result in a relatively slowly decaying forward shock, and later limits require an early jet break $t_{\mathrm{j}} \sim 0.2 \mathrm{~d}$. The detailed analysis of this problem is beyond the scope of this letter.

Progress in further study of the particular case of GRB 060117 depends on the measurement of its distance. 
Therefore the follow-up and identification of host galaxy with a large telescope is of high importance.

A larger sample of GRB rapid follow-ups is needed to decide whether this kind of transition, already suggested for other bursts, is common.

Acknowledgements. The telescope FRAM was built and is operated under the support of the Czech Ministry of Education, Youth, and Sports through its grant programs LA134 and LC527.

M.J. would like to thank to the Spanish Ministry of Education and Science for the support via grants AP2003-1407, ESP2002-04124-C03-01, and AYA2004-01515 (+ FEDER funds), M.P. was supported by the Grant Agency of the Academy of Sciences of the Czech Republic grant B300100502. The currently used FRAM wide-field camera was obtained with support from ESA PECS Project 98023.

We would also like to thank Primo Vitale for care for the telescope, Rene Goerlich for his help with Gemini GOTO and Petr Heinzel, the director of the Astronomical Institute of the Academy of Sciences of the Czech Republic, for his generous support during the telescope testing at the site of the Astronomical Institute in Ondřejov.

\section{References}

Akerlof, C., Balsano, R., Barthelmy, S., et al. 1999, Nature, 398, 400 Barthelmy, S. D., Butterworth, P., Cline, T. L., et al. 1995, Ap\&SS, 231, 235 Björnsson, G., Gudmundsson, E. H., \& Jóhannesson, G. 2004, ApJ, 615, L77 Blustin, A., Band, D., Barthelmy, S., et al. 2006, ApJ, 637, 901 Burrows, D. N., Hill, J. E., Nousek, J. A., et al. 2005, Space Sci. Rev., 120, 165 Campana, S., Barthelmy, S., Gehrels, N., et al. 2006, GCN, 4533

Chincarini, G., Moretti, A., Romano, P., et al. 2005 [arXiv: astro-ph/0506453], 1
Cummings, J., Barbier, L., Barthelmy, S., et al. 2006, GCN, 4538

de Ugarte Postigo, A., Jelínek, M., Gorosabel, J., et al. 2005, in Astrofísica Robótica en España, ed. A. Castro-Tirado, B. de la Morena, \& J. Torres, Madrid, 35

Golenetskii, S., Aptekar, R., Mazets, E., et al. 2006, GCN, 4542

Guidorzi, C., Monfardini, A., Gomboc, A., et al. 2005, ApJ, 630, L121

Jelínek, M., Kubánek, P., Prouza, M., Nekola, M., \& Hudec, R. 2006, GCN, 4536 Kubánek, P., Jelínek, M., Nekola, M., et al. 2004, in AIP Conf. Proc., 727, Gamma-Ray Bursts: 30 Years of Discovery

Kubánek, P., Jelínek, M., Prouza, M., Nekola, M., \& Hudec, R. 2006, GCN, 4535 Lazzati, D., Rossi, E., Covino, S., Ghisellini, G., \& Malesani, D. 2002, A\&A, 396, L5

Li, W., Filippenko, A. V., Chornock, R., \& Jha, S. 2003, ApJ, 586, L9

Monet, D. B. A., Canzian, B., Dahn, C., et al. 1998, VizieR Online Data Catalog, 1252,0

Nysewander, M., LaCluyze, A., Reichart, D., et al. 2006, GCN, 4548

Pelangeon, A., \& Atteia, J. L. 2006, GCN, 4544

Pierre Auger Collaboration 2005, in Proceedings of the 29th International Cosmic Ray Conference, Pune, India, also the FERMILAB preprint FERMILAB-PUB-05-469-A-TD

Piran, T. 1999, Phys. Rep., 314, 575

Rykoff, E., Mangano, V., Yost, S., et al. 2006, ApJ, 638, L5

Sari, R., \& Piran, T. 1999, ApJ, 520, 641

Schlegel, D., Finkbeiner, D., \& Davis, M. 1998, ApJ, 500, 525

Schmidt, B., Wieringa, M., Frail, D. A., \& Soderberg, A. 2006, GCN, 4546

Shao, L., \& Dai, Z. G. 2005, ApJ, 633, 1027

Shen, R., Kumar, P., \& Robinson, E. 2005 [arXiv: astro-ph/0512489]

Šimon, V., Hudec, R., Masetti, N., \& Pizzichini, G. 2001, in ESA SP-459:

Exploring the Gamma-Ray Universe, 441

Tanvir, N. 2006, GCN, 4534

Terada, Y., Yamaoka, K., Sugita, S., et al. 2006, GCN, 4573

Zhang, B., Kobayashi, S., \& Mészáros, P. 2003, ApJ, 595, 950 